\title{
Spatiotemporal Organization of Correlated Local Activity within Global Avalanches in Slowly Driven Interfaces
}

\author{
Ramon Planet, ${ }^{1,2,3}$ Juan M. López, ${ }^{4}$ Stéphane Santucci, ${ }^{5,6}$ and Jordi Ortín ${ }^{1,2}$ \\ ${ }^{1}$ Departament de Física de la Matèria Condensada, Universitat de Barcelona, Martí $i$ Franquès 1, 08028 Barcelona, Spain \\ ${ }^{2}$ Universitat de Barcelona Institute of Complex Systems, Barcelona, Spain \\ ${ }^{3}$ Institut Lumière Matière, UMR 5306 Université Lyon 1-CNRS, Université de Lyon, 69622 Villeurbanne, France \\ ${ }^{4}$ Instituto de Física de Cantabria (IFCA), CSIC-Universidad de Cantabria, E-39005 Santander, Spain \\ ${ }^{5}$ Laboratoire de physique, CNRS UMR 5672, Ecole Normale Supérieure de Lyon, \\ 46 Allée d'Italie, 69364 Lyon Cedex 07, France \\ ${ }^{6}$ Lavrentyev Institute of Hydrodynamics, Novosibirsk, Russia
}

(Received 23 October 2017; published 18 July 2018)

\begin{abstract}
We study the jerky response of slowly driven fronts in disordered media, just above the depinning transition. We focus on how spatially disconnected clusters of internally correlated activity lead to largescale velocity fluctuations in the form of global avalanches and identify three different ways in which local activity clusters may organize within a global avalanche, depending on the distance to criticality. Our analysis provides new scaling relations between the power-law exponents of the statistical distributions of sizes and durations of local bursts and global avalanches. Fluid fronts of imbibition in heterogeneous media are taken as a case study to validate these scaling relations.
\end{abstract}

DOI: 10.1103/PhysRevLett.121.034101

Under a slow external driving, out-of-equilibrium heterogeneous systems can respond in the form of collective excitations $[1,2]$. This phenomenon covers a wide range of length scales from a few nanometers or millimeters, as in magnetic domain walls [3], confined magnetic plasmas $[4,5]$, rock and wood compression [6,7], and yielding of amorphous materials [8], all the way up to geological scales for earthquakes $[9,10]$. These avalanches are usually measured in the time evolution of a global, bulk-averaged quantity, often referred to as crackling noise [2]. For critical or near-critical systems, avalanches obey power-law probability distributions lacking a characteristic scale. Ideally, the goal is to extract information concerning the local dynamics, effective transport coefficients, relevant nonlinearities, and interactions from analyzing the avalanches in the global observable. However, such a task is generally difficult, since the global dynamics involves local simultaneous avalanches, which could even be correlated, occurring at distant places in the system [11,12]. Therefore, studying avalanches in the local activity would be generally preferred since they are directly connected to the actual microscopic dynamics [13]. Interestingly, experiments on fracture fronts [12,14] and fluid imbibition [15] have been able to track local space-time activity.

In this Letter, we provide an analysis of the dynamics of slowly driven fronts near the critical depinning transition. Our goal is to relate local activity clusters to bursts in the global velocity time series. We characterize how local activity organizes spatiotemporally during a global burst depending on the distance to the critical depinning transition.
New scaling relations connecting sizes and durations of avalanches at both description levels are, thus, established.

We consider an interfacial front $h(x, t)$ moving through a two-dimensional disordered medium under the action of an external driving force. We assume that the front moves with a small but finite average velocity $\bar{v}$. A site $x$ is said to be active when its instantaneous local velocity $\Delta h(x, t) / \Delta t$ exceeds a prescribed (arbitrary) threshold $v_{\text {th }}$, providing a separation between local burst motion and the imposed front advancement. A local activity cluster is a spatially connected cluster of active sites. The size $A$ of the cluster is defined as $A=\int_{x_{0}}^{x_{0}+L_{x}} d x \int_{y_{0}}^{y_{0}+L_{y}} d y \Theta\left(v(x, y)-v_{\text {th }}\right)$, where $v(x, y)$ is the local velocity field of the front, $L_{x}$ and $L_{y}$ are the lateral dimensions of the bounding box of the corresponding local cluster, and $\Theta$ is the step function. The duration $D$ of the local activity cluster is the time spent by the front to go through the cluster. Near the critical depinning point $\bar{v}=0$, the average size $\langle A\rangle$ generically scales as $\sim D^{\gamma a}$.

Most experimental systems do not allow direct measurement of the local activity of driven fronts. Instead, a global signal $V(t, \ell)=(1 / \ell) \int_{0}^{\ell} d x v(x, t)$ is accessible. If collective motion takes place in the system, this reflects in a jerky signal in time, as shown, for instance, in Fig. 1(b) for imbibition experiments.

Slightly above the critical point there is local activity at nearly all times, and, thus, $V(t, \ell)$ is nearly always above 0 . A finite detection threshold $V_{\text {th }}$ is necessary to unambiguously define avalanches of the global signal and to 


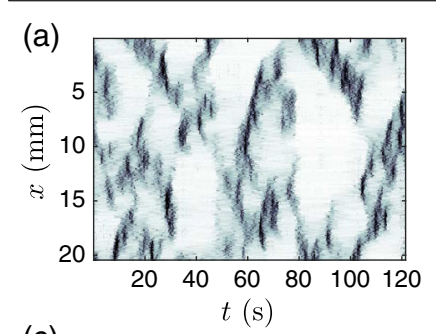

(c)
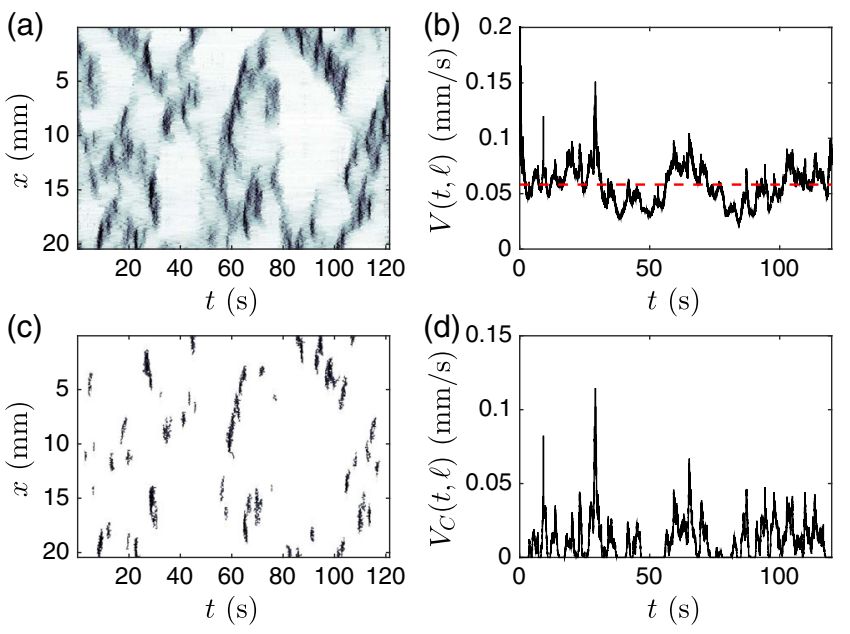

FIG. 1. (a) Activity map of local front velocities in an imbibition displacement with injection velocity $\bar{v}=0.057 \mathrm{~mm} / \mathrm{s}$ in an observation window of lateral size $\ell=20 \mathrm{~mm}$. (b) Global velocity signal obtained from integrating this local activity map with respect to $x$. The discontinuous line is the mean velocity. (c) Activity map of local velocities clipped with $v_{\text {th }}=3 \bar{v}$. (d) Corresponding global signal obtained from integrating this clipped activity map with respect to $x$.

measure their duration $T$ and size $S$. These properties are given, respectively, by the time interval elapsed in an excursion of $V(t, \ell)$ above $V_{\text {th }}$ and by the area enclosed in the excursion, $S=\int_{t_{0}}^{t_{0}+T} d t\left[V(t, \ell)-V_{\text {th }}\right]$, where $t_{0}$ is the time of the first threshold crossing. Average avalanche sizes scale as $\langle S\rangle \sim T^{\gamma}$ with their durations. This scaling relation is valid even for noncritical systems [16]. Near depinning, the sizes and durations of both local activity clusters and global events take values in a very broad range. Their statistical distributions are usually written as a power law with an exponential cutoff $\mathcal{P}(X) \sim X^{-\tau_{X}} \exp \left(-X / \xi_{X}\right)$. The critical exponent $\tau_{X}$ reflects scale invariance in a wide range of $X$ below the upper cutoff $\xi_{X}[15,17,18]$. Both $\tau_{X}$ and $\xi_{X}$ will, in general, be different for local activity clusters and global avalanches.

Thresholding plays a key role in the analysis of avalanche statistics $[19,20]$. Most existing studies typically analyze local activity clusters and global avalanches separately, applying independent thresholds. In order to make a connection between global avalanche statistics and local activity, however, the thresholds applied cannot be chosen independently. We propose here a new approach based on a single threshold, $v_{\text {th }}$. First, we generate the activity map of local velocities $v(x, t)$ from experiment [Fig. 1(a)] and apply to it the arbitrary threshold $v_{\text {th }}$, such that a clipped activity map $v_{C}(x, t)$ is obtained [Fig. 1(c)]. $v_{C}(x, t)=$ $v(x, t)$ if $v(x, t) \geq v_{\text {th }}$ and $v_{C}(x, t)=0$ otherwise. Local activity clusters are defined now in the usual way, using $v_{C}(x, t)$ instead of $v(x, t)$. The corresponding global velocity $V_{C}(t, \ell)$ is obtained by averaging the clipped activity map over a window of lateral size $\ell$. This signal returns intermittently to zero [Fig. 1(d)], and no further thresholding is needed. Global avalanches are excursions of this signal between consecutive zero values. We expect that the new global signal $V_{C}(t, \ell)$ will exhibit the same statistical features than the original signal $V(t, \ell)$ above an arbitrary (judicious) threshold. In imbibition experiments, we have verified indeed that the probability density functions (PDFs) of $S$ and $T$ are well approximated by power laws with exponential cutoffs in both cases and also that the values of the corresponding power-law exponents are the same within experimental uncertainty.

We can relate now local activity clusters to global avalanches. With the definition of $V_{C}(t, \ell)$, the size of a global avalanche of duration $T$ is the sum of the number of local events during its occurrence:

$$
S(T)=\frac{1}{\ell} \sum_{j \mid D_{j} \in T} A_{j}=\frac{N(T)}{\ell}\langle A\rangle,
$$

where $N(T)$ is the mean number of local events in a global avalanche of duration $T$, and $\langle\cdots\rangle$ is the average size (or duration) of the local events within the global avalanche [21]. Assuming that the scaling relation $A \sim D^{\gamma a}$ applies to all local avalanches contributing to a global event of duration $T$, Eq. (1) can be rewritten in the continuum limit as

$$
S(T) \sim \frac{N(T)}{\ell} \int_{0}^{T} d D D^{\gamma_{a}} \mathcal{P}(D ;\{D \in T\}),
$$

where $\mathcal{P}(D ;\{D \in T\})$ is the PDF of durations of the local avalanches in a global avalanche of duration $T$ [21]. Equation (2) is general and can be used in different scenarios depending on $\mathcal{P}(D ;\{D \in T\})$. We can identify three different arrangements of local bursts within a global avalanche depending on how close the system is to criticality.

Arrangement I: Static limit.—Right at the critical pinning point $\bar{v}=0$, and the lateral correlation length of the front fluctuations diverges, $\ell_{c} \rightarrow \infty$. Interfacial motion is fully correlated so that advances of the front take place by single local clusters of activity. In this situation, there is a one-toone correspondence between local and global activity. Equation (1) adopts the form $S(T)=A(T) / \ell$, and the relation $\gamma=\gamma_{a}$ follows straightforwardly.

Arrangement II: Simultaneous local avalanches.-When the correlation length of interfacial fluctuations does not diverge but it is a non-negligible fraction of the system size, the local activity is highly heterogeneous and moving sites coexist with large pinned regions. Any given site goes through random time intervals of activity separated by long intervals of calm while activity is localized elsewhere. When time intervals of activity and calm are uncorrelated, i.e., if local activity follows a renewal process [27], then accumulation of short-lived local activity clusters will not lead to macroscopic collective events on much longer 
timescales. This leads to the lowest-order approximation that global avalanches are dominated by local events of the same duration, while shorter local events occurring within the same time window only affect the variance of $S(T)$ and not the average. Thus, a global velocity fluctuation that lasts for a time $T$ corresponds on average to a number $N(T)$ of coexisting, spatially separated, local avalanches of the same duration, i.e., $D=T$. Mathematically, this translates to a case where $\mathcal{P}(D ;\{D \in T\})$ becomes a delta function $\delta(D-T)$. Introducing it in Eq. (2), we obtain $S(T) \sim N(T) T^{\gamma a} \sim T^{\gamma}$.

In order to find a relation between $\gamma_{a}$ and $\gamma$, an estimate of $N$ is required. The morphological properties of the interface (roughness) can be used for this purpose. A fluctuation of the global velocity that lasts a time $T$ produces an interface fluctuation of the order of the global interface width $W(T) \sim T^{\alpha / z}$ [28]. Similarly, a single local excitation leads to an interface fluctuation of order $w(T) \sim T^{\alpha_{\mathrm{loc}} / z_{a}}$ in the same time interval. $\alpha$ and $\alpha_{\mathrm{loc}}$ are the global and local roughness exponents, and $z$ and $z_{a}$ are the global and local dynamic exponents, respectively [29]. The global surface fluctuation corresponds to the sum over all local excitations $W(T) \sim N w(T)$ so that $N \sim T^{\alpha / z-\alpha_{\mathrm{loc}} / z_{a}}$. Thus,

$$
\gamma-\frac{\alpha}{z}=\gamma_{a}-\frac{\alpha_{\mathrm{loc}}}{z_{a}} .
$$

This scaling relation connects the avalanche exponents $\gamma$ and $\gamma_{a}$ with the kinetic roughening exponents of the moving front. This is one of the main theoretical results of this Letter.

The argument can be extended to the relation between the statistics of global excitations and local activity clusters. If $\mathcal{P}_{S}(S)$ is the PDF of avalanche sizes and $\mathcal{P}_{A}(A)$ is the PDF of local activity areas, we have that $\mathcal{P}_{S}(S) d S=\mathcal{P}_{A}(A) d A$. It follows that

$$
\tau_{A}=1+\frac{\gamma}{\gamma_{a}}\left(\tau_{S}-1\right),
$$

which connects statistical and dynamic properties of local activity and global velocity avalanches. Assuming an uncorrelated process, a particular instance of this scaling relation for $N \sim S^{\mu}$ with $\mu=1 / 2$ was obtained and confirmed by numerical simulations for interfacial crack dynamics [11]. According to our analysis, the exponent $\mu$ is given by $1-\gamma_{a} / \gamma$ in general.

Arrangement III: Random occurrence of local activity clusters.-When the correlation length becomes much smaller than the lateral size of the observation window $\ell_{c} \ll \ell$, occurrences of activity in a global avalanche can be regarded as independent events. In this case, in an avalanche of duration $T$, local activity clusters with duration $D<T$ can occur independently from each other with equal probability so that $T \sim N(T)$. Now, $N$ bears no relation with the morphology of the front. During larger global avalanches, the duration of the corresponding activity clusters is limited by a constant cutoff value $\xi_{D}$ [15]. If we assume again in this case that the statistics of global avalanches is dominated by the local events of larger duration, $\mathcal{P}(D ;\{D \in T\})=\delta\left(D-\xi_{D}\right)$. Introducing this PDF in Eq. (2), we obtain $S(T) \sim N \sim T$. This means that $\gamma=1$ independent from the value of $\gamma_{a}$.

We take imbibition fronts in disordered media as a case study to test our scaling theory above. Fluid fronts in stable imbibition displacements develop long-range lateral correlations spontaneously up to a characteristic length $\ell_{c}$ that can be tuned by the average front velocity [17]. The system has correlated activity at short length scales (below $\ell_{c}$ ) and uncorrelated motion at longer length scales. The different arrangements discussed before can be explored. Figure 1(a) shows an example of the activity map of a stable displacement in a model open fracture [15,17]. Following Ref. [15], we take $v_{\text {th }}=C \bar{v}$, where $C$ is an arbitrary clip level between 1.4 and 3 , and $\bar{v}$ is the injection velocity. We have checked that the statistical properties of local clusters are robust in the range of thresholds applied here [15]. We focus on cases where $\ell_{c}$ is always smaller than the observation window $\ell$. For this reason, we take $\ell=20 \mathrm{~mm}$. Finally, we discard tiny local avalanches of sizes smaller than the disorder patches in the cell and avalanches that are cut by the observation window [30].

In order to identify the three different arrangements discussed earlier, we characterize how local activity clusters are distributed in space and time during a global avalanche. There are two limiting cases. One is that local activity clusters occur simultaneously during a global avalanche, and then $T=\max \left(D_{i}\right)$ (top left, Fig. 2), and the other is that local avalanches occur successively inside a global avalanche (pileup), and then $T=\sum_{i} D_{i}$ (top right, Fig. 2). The bottom graph in Fig. 2 shows the ratios $\max \left(D_{i}\right) / T$ and $\sum_{i} D_{i} / T$ computed from our experimental data for a given clip level and different injection velocities. Durations are measured in units of $\xi_{T} \sim \ell_{d} / \bar{v}$, where $\ell_{d}=0.6 \mathrm{~mm}$ is the characteristic length of the disorder patches in the experimental cell [31].

The experimental results reveal indeed three different arrangements coexisting in a single imbibition displacement.

Arrangement I.-The two first leftmost experimental points in Fig. $2\left(T \bar{v} / \ell_{d} \simeq 0.05\right)$ correspond to the (critical) case where a single local cluster of activity occurs during an avalanche so that $N=1$. In this case, $\max \left(D_{i}\right) / T$ and $\sum_{i} D_{i} / T$ coincide because the two limiting cases are the same. The statistics of these events, however, is severely limited by the fact that their durations are close to the temporal experimental resolution.

Arrangement II.-Then, for avalanches in the range $0.05 \lesssim T \bar{v} / \ell_{d} \lesssim 0.2$, we observe in Fig. 2 that $\max \left(D_{i}\right) / T \simeq 1$ while $\sum_{i} D_{i} / T$ departs from unity. This is a signature that local activity clusters occur simultaneously during a global avalanche. 

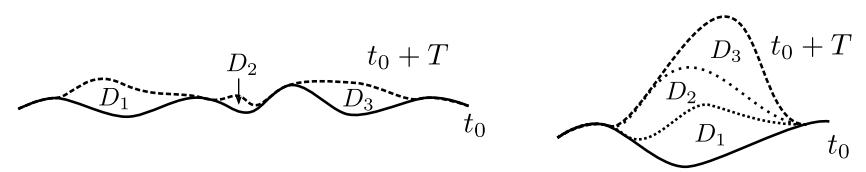

$T=\max \left(D_{i}\right)$

$$
T=\sum_{i} D_{i}
$$

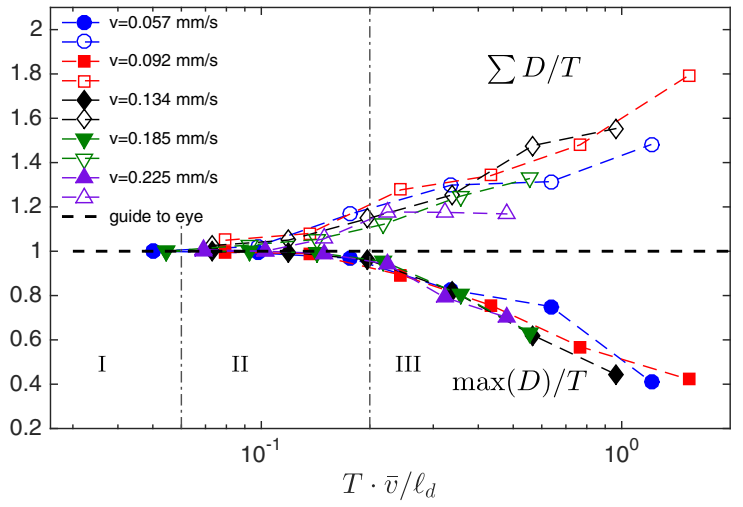

FIG. 2. Top: Scheme of horizontal (left) and vertical (right) distributions of local activity clusters. Bottom: Ratios $\max \left(D_{i}\right) / T$ (filled symbols) and $\sum_{i} D_{i} / T$ (open symbols) for a given clip $C=3$ and different injection velocities $\bar{v}$ as a function of $T \bar{v} / \ell_{d}$.

In this arrangement, we expect a scaling $N \sim T^{\alpha / z-\alpha_{10 c} / z_{a}}$. Using the values of the exponents collected in Table I, this scaling predicts that $N$ should decrease with $T$. This predicted scaling, however, is not accessible experimentally. Figure 3 shows indeed that arrangement II corresponds only to the leftmost solid symbol for which the number of local activity clusters within a global avalanche is very small and the range of dimensionless durations $T \bar{v} / \ell_{d}$ is rather limited.

Nevertheless, for this arrangement, the sizes and durations of local and global events are power-law distributed

TABLE I. Critical exponents of size and size-duration distributions of local bursts and global avalanches and kinetic roughening exponents of imbibition fronts driven at constant injection velocity in the regime of capillary disorder. Values derived from theoretical arguments are given without uncertainty.

\begin{tabular}{lcccc}
\hline \hline & & Experiments & Phase-field simulations \\
\hline & $\ell_{c} / L$ & $0.04-0.12$ & 0.1 & $\ldots$ \\
\hline Avalanche dynamics & Refs. [15,17] & Refs. [18,29,32] Ref. [33] \\
\hline $\mathcal{P}_{S}(S) \sim S^{-\tau_{S}}$ & $\tau_{S}$ & $1.00(15)$ & $1.03(6)$ & $\ldots$ \\
$\mathcal{P}_{A}(A) \sim A^{-\tau_{A}}$ & $\tau_{A}$ & $1.08(5)$ & $1.54(5)$ & $\ldots$ \\
$S \sim T^{\gamma}$ & $\gamma$ & $1.33(12)$ & $1.30(5)$ & 1.33 \\
$A \sim D^{\gamma}$ & $\gamma_{a}$ & $1.6(2)$ & $1.28(4)$ & $\ldots$ \\
$\ell \sim t^{1 / z_{a}}$ & $z_{a}$ & $1.1(1)$ & $1.59(15)$ & $\ldots$ \\
\hline Roughening kinetics & This work [34] & Refs. [18,29] & Ref. [33] \\
\hline$\omega(\ell, t) \sim \ell^{\alpha_{\text {loc }}}$ & $\alpha_{\text {loc }}$ & $0.82(5)$ & 1 & 1 \\
$\omega(L, t) \sim t^{\alpha / z}$ & $\alpha / z$ & $0.42(2)$ & $1.33 / 3$ & $\ldots$ \\
$\ell$ & $z$ & $3.0(2)$ & 3 & $\ldots$ \\
\hline \hline
\end{tabular}

over a very wide range. The values of the corresponding scaling exponents $\tau_{S}, \tau_{A}, \gamma, \gamma_{a}$, and $z_{a}$ have been reported already in Refs. [15,17]. These values are collected in Table I. We can now check the validity of our scaling relations (3) and (4). The experiments reported in Table I have provided $\gamma=1.33(12)$, in very good agreement with the value 1.30(3) obtained from Eq. (3) with the experimentally measured values of $\gamma_{a}, z_{a}$, and $\alpha / z, \alpha_{\text {loc }}$ reported in Table I. We find that Eq. (3) is reasonably satisfied. Similarly, Eq. (4) is compatible with the measured exponents. Replacing $\tau_{S}, \gamma_{a}$, and $\gamma$ measured in the experiments, Eq. (4) predicts $\tau_{A}=1.00(14)$ which compares well with the experimental value $\tau_{A}=1.08(5)$.

The scaling exponents obtained from phase-field simulations of constant flow rate imbibition displacements carried out by two different groups $[18,29,32,33]$ are also reported in Table I. These numerical results are in excellent agreement with the experimental values for the global avalanche exponents $\gamma$ and $\tau_{S}$ and for the roughening exponents $\alpha, z$, and $\alpha_{\mathrm{loc}}$. However, the local avalanche exponent $\gamma_{a}=1.28(4)$ [18] clearly differs from the experimental value 1.6(2) [15], though it is very close to the numerical estimate for the corresponding exponent of the global avalanches, $\gamma=1.30(5)$. The fact that $\gamma \simeq \gamma_{a}$ but $\tau_{A} \neq \tau_{S}$ strongly suggests that the scaling law $A \sim D^{\gamma_{a}}$ in the numerical study [18] was dominated by large avalanches $S \geq \xi_{S}$ corresponding to arrangement III, and, therefore, $\gamma$ and $\gamma_{a}$ are independent.

Arrangement III.-Finally, for global velocity avalanches of duration $T \bar{v} / \ell_{d}>0.2$, we observe a mixed arrangement where activity clusters occur simultaneously and also pile up, so this activity is off critical. We have verified that this behavior is independent of the clip level. In this arrangement, a global avalanche is the superposition of many independent bursts of local activity. Global avalanches have durations $T$ whose $\mathcal{P}(T)$ is influenced by the upper cutoff $\xi_{T}$. We expect that $N$ scales linearly with $T$. This behavior is verified in Fig. 3. The prediction $S \sim T$ for this arrangement is compatible with the tendency

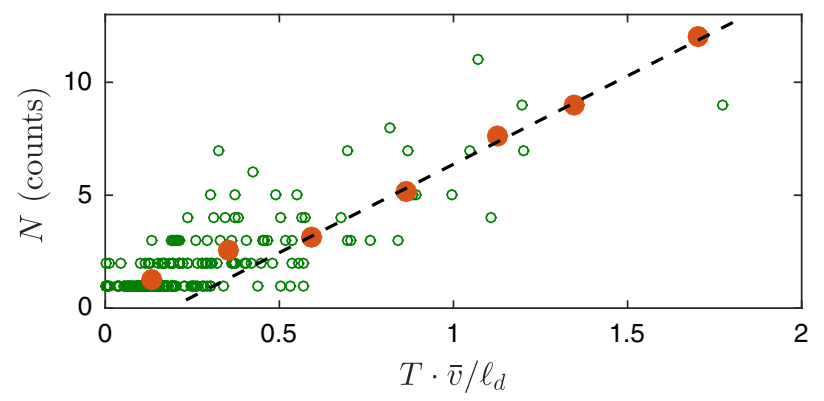

FIG. 3. Total number of local events that occur in a global avalanche of duration $T$ as a function of $T \bar{v} / \ell_{d}$. Data from experiments at $\bar{v}=0.057 \mathrm{~mm} / \mathrm{s}$ with $C=3$. Open dots are individual global avalanches and solid dots their average in equally spaced bins. The dashed line is a guide to the eye. 
of the crest of the joint probability distribution $\mathcal{P}(S, T)$ for $T>2$ reported in the right panel of Fig. 17 in Ref. [31].

To conclude, we have presented an analysis of how global velocity fluctuations build up from local activity clusters in dynamic growth processes. Consistent thresholds for local bursts and global avalanches, as proposed here, must be used. We have identified three possibilities. A first arrangement (I) in which each short-lived global avalanche results from a single local activity cluster. This would be the only possible scenario at criticality. In contrast, global avalanches of longer duration are composed of spatially disconnected, internally correlated local clusters. The specific way in which they organize in space and time within a global avalanche depends strongly on the distance to criticality. Local bursts can occur simultaneously within a global avalanche (II) or randomly in space and time (III). Analyzing the interplay between front dynamics and morphology in arrangement II and using simple scaling arguments, we have derived new scaling relations [Eqs. (3) and (4)] between kinetic roughening exponents and the power-law exponents of both local activity and global velocity avalanches. We have used fluid imbibition displacements to validate the proposed scaling relations. Our arguments, however, are very general, and, thus, we expect our results to be applicable to a wide range of slowly driven interfacial systems where surface roughness fluctuations are produced by avalanches of local motion.

The work on avalanches in Barcelona is partially funded by AGAUR (Generalitat de Catalunya) through Project No. 2017 SGR 1061 and by MINECO (Spain) through Projects No. MAT2015-69-777-REDT and No. FIS201678507-C2-2-P. R.P. is supported by the Secretary for Universities and Research of the Ministry of Economy and Knowledge of the Government of Catalonia and the COFUND program of the Marie Curie Actions of the 7th R\&D Framework Programme of the European Union (Contract No. 2014 BP-B 00138). R. P. also acknowledges support from the AXA Research Fund. J. M. L. acknowledges financial support from MINECO (Spain) through Grant No. FIS2016-74957-P. Finally, S. S. acknowledges financial support from the Russian Government through Grant No. n.14.W03.31.0002.

[1] Avalanches in Functional Materials and Geophysics, Understanding Complex Systems, edited by E. K. Salje, A. Saxena, and A. Planes (Springer, Cham, 2017).

[2] J. P. Sethna, K. A. Dahmen, and C. R. Myers, Crackling noise, Nature (London) 410, 242 (2001).

[3] G. Durin, F. Bohn, M. A. Corrêa, R. L. Sommer, P. Le Doussal, and K. J. Wiese, Quantitative Scaling of Magnetic Avalanches, Phys. Rev. Lett. 117, 087201 (2016).

[4] R. Sánchez, B. P. van Milligen, D. E. Newman, and B. A. Carreras, Quiet-Time Statistics of Electrostatic Turbulent
Fluxes from the JET Tokamak and the W7-AS and TJ-II Stellarators, Phys. Rev. Lett. 90, 185005 (2003).

[5] O. Castellanos, J. M. López, J. M. Sentíes, and E. Anabitarte, Intermittency, avalanche statistics, and long-term correlations in a turbulent plasma, J. Stat. Mech. (2013) P04022.

[6] J. Baró, A. Corral, X. Illa, A. Planes, E. K. H. Salje, W. Schranz, D. E. Soto-Parra, and E. Vives, Statistical Similarity between the Compression of a Porous Material and Earthquakes, Phys. Rev. Lett. 110, 088702 (2013).

[7] T. Mäkinen, A. Miksic, M. Ovaska, and M. J. Alava, Avalanches in Wood Compression, Phys. Rev. Lett. 115, 055501 (2015).

[8] C. Liu, E. E. Ferrero, F. Puosi, J.-L. Barrat, and K. Martens, Driving Rate Dependence of Avalanche Statistics and Shapes at the Yielding Transition, Phys. Rev. Lett. 116, 065501 (2016).

[9] D. Schorlemmer, S. Wiemer, and M. Wyss, Variations in earthquake-size distribution across different stress regimes, Nature (London) 437, 539 (2005).

[10] W. Klein, H. Gould, K. F. Tiampo, J. B. Silva, T. Gu, J. Kazemian, C. Serino, and J. B. Rundle, Avalanches in Functional Materials and Geophysics, Understanding Complex Systems, edited by E. K. Salje, A. Saxena, and A. Planes (Springer, Cham, 2017), pp. 1-18.

[11] L. Laurson, S. Santucci, and S. Zapperi, Avalanches and clusters in planar crack front propagation, Phys. Rev. E 81, 046116 (2010).

[12] K. T. Tallakstad, R. Toussaint, S. Santucci, and K. J. Måløy, Non-Gaussian Nature of Fracture and the Survival of Fat-Tail Exponents, Phys. Rev. Lett. 110, 145501 (2013).

[13] L. Laurson and M. Alava, Local waiting times in critical systems, Eur. Phys. J. B 42, 407 (2004).

[14] K. J. Måløy, S. Santucci, J. Schmittbuhl, and R. Toussaint, Local Waiting Time Fluctuations along a Randomly Pinned Crack Front, Phys. Rev. Lett. 96, 045501 (2006).

[15] S. Santucci, R. Planet, K. J. Måløy, and J. Ortín, Avalanches of imbibition fronts: Towards critical pinning, Europhys. Lett. 94, 46005 (2011).

[16] S. Redner, A Guide to First-Passage Processes (Cambridge University Press, Cambridge, England, 2001).

[17] R. Planet, S. Santucci, and J. Ortín, Avalanches and NonGaussian Fluctuations of the Global Velocity of Imbibition Fronts, Phys. Rev. Lett. 102, 094502 (2009).

[18] M. Pradas, J. M. López, and A. Hernández-Machado, Avalanche dynamics in fluid imbibition near the depinning transition, Phys. Rev. E 80, 050101 (2009).

[19] F. Font-Clos, G. Pruessner, N. R. Moloney, and A. Deluca, The perils of thresholding, New J. Phys. 17, 043066 (2015).

[20] S. Janićević, L. Laurson, K. J. Måløy, S. Santucci, and M. J. Alava, Interevent Correlations from Avalanches Hiding Below the Detection Threshold, Phys. Rev. Lett. 117, 230601 (2016).

[21] See the Supplemental Material at http://link.aps.org/ supplemental/10.1103/PhysRevLett.121.034101 for the derivation, which includes Refs. [22-26].

[22] J. Soriano, J. Ortín, and A. Hernández-Machado, Experiments of interfacial roughening in Hele-Shaw flows with weak quenched disorder, Phys. Rev. E 66, 031603 (2002). 
[23] J. J. Ramasco, J. M. López, and M. A. Rodríguez, Generic Dynamic Scaling in Kinetic Roughening, Phys. Rev. Lett. 84, 2199 (2000).

[24] A. Hernández-Machado, J. Soriano, A. M. Lacasta, M. A. Rodríguez, L. Ramírez-Piscina, and J. Ortín, Interface roughening in Hele-Shaw flows with quenched disorder: Experimental and theoretical results, Europhys. Lett. 55, 194 (2001).

[25] E. Pauné and J. Casademunt, Kinetic Roughening in TwoPhase Fluid Flow through a Random Hele-Shaw Cell, Phys. Rev. Lett. 90, 144504 (2003).

[26] R. Planet, M. Pradas, A. Hernández-Machado, and J. Ortín, Pressure-dependent scaling scenarios in experiments of spontaneous imbibition, Phys. Rev. E 76, 056312 (2007).

[27] S. B. Lowen and M. C. Teich, Fractal renewal processes generate $1 / f$ noise, Phys. Rev. E 47, 992 (1993).

[28] A.-L. Barabási and H. E. Stanley, Fractal Concepts in Surface Growth (Cambridge University Press, Cambridge, England, 1995), p. 386.
[29] J. M. López, M. Pradas, and A. Hernández-Machado, Activity statistics, avalanche kinetics, and velocity correlations in surface growth, Phys. Rev. E 82, 031127 (2010).

[30] Y.-J. Chen, S. Papanikolaou, J. P. Sethna, S. Zapperi, and G. Durin, Avalanche spatial structure and multivariable scaling functions: Sizes, heights, widths, and views through windows, Phys. Rev. E 84, 061103 (2011).

[31] X. Clotet, S. Santucci, and J. Ortín, Experimental study of stable imbibition displacements in a model open fracture. II. Scale-dependent avalanche dynamics, Phys. Rev. E 93, 012150 (2016).

[32] M. Pradas, Interfaces in disordered media: Scaling growth, avalanche dynamics, and microfluidic fronts," Ph.D. thesis, Universidad de Barcelona, 2009.

[33] M. Rost, L. Laurson, M. Dubé, and M. Alava, Fluctuations in Fluid Invasion into Disordered Media, Phys. Rev. Lett. 98, 054502 (2007).

[34] Kinetic roughening exponents of the moving fronts have been measured specifically for the present work. The experimental results are reported in the Supplemental Material [21]. 